\section{Modifizierte Allergenextrakte sind sicher}

\section{Bei der subkutanen spezifischen Immuntherapie kommen neben nativen Extrakten auch Allergoide zum Einsatz. In einer Phase-IV- Studie wurde die Verträglichkeit eines Glutaraldehyd-modifizierten, depigmentierten Allergenextrakts untersucht.}

\begin{abstract}
C panische Allergologen bezogen in ihre prospektive multizentrische Studie 766 Patienten mit Rhinokonjunktivitis und/oder Asthma aufgrund einer Sensibilisierung gegen Hausstaubmilben und/oder Pollen ein. Ihnen wurde ein polymerisierter, depigmentierter und an Aluminiumhydroxid adsorbierter Allergenextrakt in einer vier- bis sechswöchigen Auftitrationsphase und dann mit monatlichen Injektionen über ein Jahr gegeben. Kein Patient erhielt vor der Injektion Antihistaminika oder Gluko-
\end{abstract}

kortikoide. Die Einteilung des Schweregrads von Nebenwirkungen erfolgte nach den Vorgaben des EAACI-Positionspapiers für die spezifische Immuntherapie.

Alle Patienten beendeten die Studie. Im Beobachtungszeitraum wurden 54 klinisch relevante Lokalreaktionen (0,4\% der Injektionen) gesehen, davon 43 vom Soforttyp und elf verzögert auftretend. Systemische Reaktionen traten 34-mal bei insgesamt zwölf Patienten auf. Sechs der Reaktionen waren Soforttypreaktionen vom Schweregrad 2 und
28 waren vom verzögerten Typ. Systemische Reaktionen vom Schweregrad 2 oder 3 zeigten sich bei $0,12 \%$ aller Reaktionen. Es kam zu keinem anaphylaktischen Schock. Alle unerwünschten Ereignisse gingen spontan ohne weitere medikamentöse Intervention zurück.

Fazit: In dieser prospektiven Beobachtungsstudie erwies sich die spezifische Immuntherapie mit modifizierten und depigmentierten Allergenen als sicher. Die Nebenwirkungsrate lag unter der einer spezifischen Immuntherapie mit nativen Allergenextrakten.

Casanovas M et al. Safety of immunotherapy with therapeutic vaccines containing depigmented and polymerized allergen extracts. Clin Exp Allergy 2007; 37: 434-40

\section{Immuntherapie mit Allergoidtabletten}

\section{Allergoide haben sich in der subkutanen Immuntherapie ihren Platz als Alternative zu nicht modifizierten Extrakten erkämpft. In einer portugiesischen Pilotstudie wurde jetzt erstmals der Einsatz von All- ergoiden in Tablettenform zur sublingualen Applikation untersucht.}

m Rahmen einer Doppelblindstudie wurden 33 Patienten mit allergischer Rhinokonjunktivitis und zum Teil auch mit allergischem Asthma aufgrund einer Sensibilisierung gegen Gräserpollen in zwei Gruppen aufgeteilt. Die erste Gruppe erhielt über zwei Jahre eine Immuntherapie mit einer mukosal zu applizierenden Tablette, die Allergoide von Holcus lanatus, Phleum pratense und Poa pratensis enthielt. Die zweite Gruppe erhielt Plazebo. Während der Pollenbenötigte Medikamente dokumentiert, nach zwei Jahren wurde ein nasaler Provokationstest durchgeführt. Die Daten von 20 Teilnehmern konnten ausgewertet werden.

Während sich in der Plazebogruppe der Symptomscore über den Studienzeitraum hinweg nicht änderte, erlebten die Teilnehmer der Verumgruppe eine signifikante Verbesserung der Symptome Rhinorrhö, Niesen und saison wurden täglich Symptome und
Konjunktivitis im zweiten gegenüber dem ersten Jahr. Nach zwei Jahren zeigte sich auch im nasalen Provokationstest in der Immuntherapiegruppe eine signifikante Reduktion der Reaktivität im Vergleich zum Ausgangswert. In beiden Interventionsjahren verrin- gerte sich der Bedarf an nasalen Glukokortikoiden in der Verumgruppe zudem signifikant.

Fazit: Die Ergebnisse dieser Studie deuten auf eine Wirksamkeit der sublingualen Immuntherapie mit Allergoiden in Tablettenform bei Gräserpollenallergie hin - bei sehr guter Verträglichkeit. $f_{k}$

Palma-Carlos AG et al. Clinical efficacy and safety of preseasonal sublingual immunotherapy with grass pollen carbamylated allergoid in rhinitic patients. A doubleblind, placebo-controlled study. Allergol Immunopathol 2006; 34: 194-8

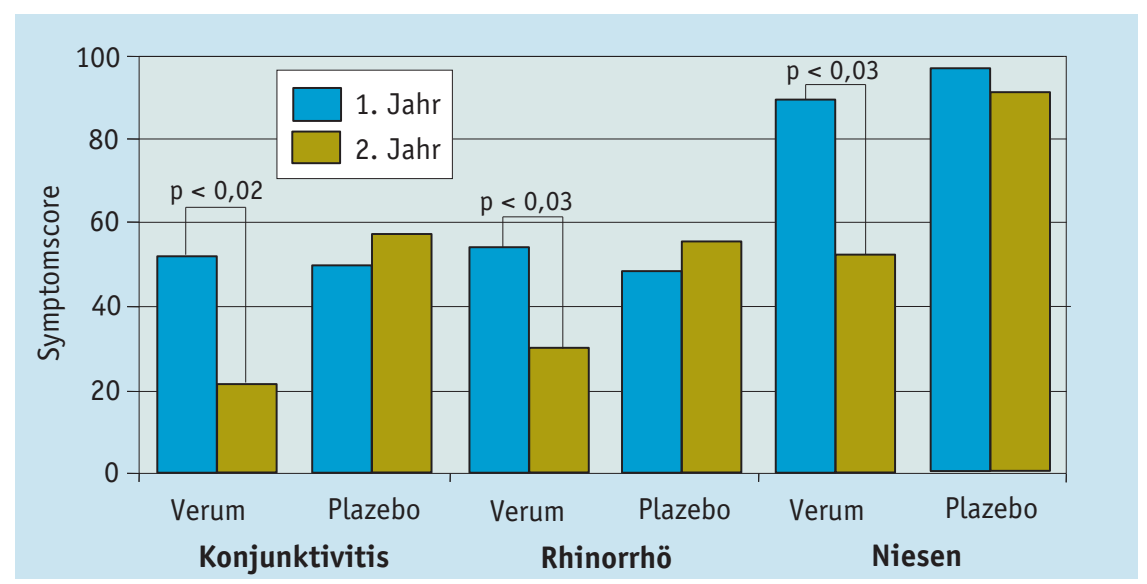

Klinischer Effekt einer sublingual applizierten Allergoidtablette 